\title{
The Effect of Curcumin Supplementation on Selected Markers of Delayed Onset Muscle Soreness (DOMS)
}

\author{
${ }^{1}$ Babak Nakhostin-Roohi ${ }^{*}{ }^{2}$ Arash Nasirvand Moradlou, ${ }^{2}$ Sahar Mahmoodi Hamidabad, \\ ${ }^{3}$ Babak Ghanivand
}

${ }^{1}$ Department of Sport Sciences, Faculty of Humanities, Ardabil Branch, Islamic Azad University, Ardabil, Iran. ${ }^{2}$ Department of Physical Education, Mohaghegh Ardabili University, Ardabil, Iran. ${ }^{3}$ Department of Sport Sciences, Faculty of Humanities, Rasht Branch, Islamic Azad University, Rasht, Iran.

\begin{abstract}
Inflammation and pain induced by delayed onset muscle soreness (DOMS) can be induced by eccentric exercise or an unaccustomed activity. The condition can causes problems in exercising and for athletes. The purpose of this study was to assess the effect of $150 \mathrm{mg}$ curcumin supplementation immediately after intensive eccentric exercise. Evaluations were made for total antioxidant Capacity (TAC), muscle damage markers, and DOMS induced pain. Ten healthy young males (age, $25.0 \pm 1.6$ years; height, $178.9 \pm 4.1 \mathrm{~cm}$; body mass, $81.1 \pm 6.8 \mathrm{~kg}$; fat $\%, 14.2 \pm 2.1$ ) completed a double blind randomized-controlled crossover trial to estimate the effects of oral curcumin supplementation (150mg) and a placebo on squat performance and DOMS following unaccustomed heavy eccentric exercise. Curcumin $(\mathrm{CU})$ or placebo $(\mathrm{P})$ was taken at the prescribed dose immediately after eccentric squat exercises; administrations were separated by a 14-day washout period. Measurements were made at the baseline, immediately, 24, 48, and 72h after exercise comprising: (a) limb pain (1-10 cm visual analogue scale; VAS), (b) total antioxidant capacity (TAC) (c) serum markers of muscle damage and inflammation. Measurements taken after exercise showed significantly reduced levels of pain, creatine kinase (CK), alanine aminotransferase (ALT), and aspartate aminotransferase (AST) in $\mathrm{C}$ group compared with group $\mathrm{P}$ group $(\mathrm{P}<0.05)$. TAC remained significantly high in group $\mathrm{C}$ after exercise compared with levels in group $\mathrm{P}(\mathrm{P}<0.05)$. The findings of this study suggest that a $150 \mathrm{mg}$ dose of curcumin may have antioxidant, anti-inflammatory and analgesic effects on DOMS.
\end{abstract}

KEY WORDS: Delayed Onset Muscle Soreness (DOMS), Curcumin, Total Antioxidant Capacity (TAC), Muscle Damage, Squat Exercises.

\section{INTRODUCTION}

Delayed onset muscle soreness (DOMS) from training or exercise produces unpleasant and undesirable feelings of pain and muscle stiffness. That. The condition can discourage a beginner or an experienced athlete from continuing with the exercise (1-4). DOMS results in discomfort at the site of the injury, inflammation, oxidative stress, edema and loss of muscle function and strength $(5,6)$. Extensive research has been done to investigate procedures to reduce DOMS and many studies have reported that nutrition interventions can reduce experiences of DOMS (7). Commonly known nutritional interventions

*. Corresponding Author:

Babak Nakhostin-Roohi

E-mail: b.nakhostinroohi@iauardabil.ac.ir 
include caffeine, omega-3 fatty acids, and taurine (8-10).

Recent research attention has focused on the effects of nutraceutical bioactive compounds such as polyphenols (11). Polyphenols are a class of organic chemical compound, mainly found in plants that are characterized by the presence of multiples of phenol structural units (12). Curcumin is a hydroxycinnamic acid derivative and its structure contains two hydrophobic polyphenolic rings with two carbonyl groups. It is the main curcuminoid found in the spice turmeric, a plant alkaloid obtained from ground rhizome of the perennial herb Curcuma longa (13). According to investigations, curcumin has multiple biofunctional activities including anti-inflammatory, antioxidant, anti-cancer, anti-diabetes and chemo preventative activities (14-18).

Evidence from tests suggest that in some conditions, curcumin possess similar antiinflammatory activity as some of the common non-steroidal anti-inflammatory drugs (NSAIDs) such as indomethacin, Vioxx, Celebrex, and ibuprofen, but without many of the side effects, such as gastrointestinal distress and cardiovascular complications (19, 20). Accordingly, some investigations have suggested that curcumin could be effective in controlling inflammation and oxidative stress induced by DOMS.

Some investigations have evaluated for the effect of curcumin on DOMS (21-24). However, to the best of our knowledge, no study to date has tested administration of $150 \mathrm{mg}$ curcumin supplement immediately after intensive eccentric exercise. Therefore, the aim of this study was to evaluate the effect of acute curcumin supplementation on the DOMS markers immediately after intensive eccentric exercise.

\section{MATERIALS AND METHODS}

Participants. 10 healthy young males gave written informed consent and took part in this study. This study was conducted according to the guidelines laid down in the Declaration of Helsinki and approved by the ethics committee of Ardabil Branch, Islamic Azad University. All volunteers completed a questionnaire on physical activity, exercise, dietary intake and health history prior to participation in the study.
Study Design. The study was designed as a double-blind randomized-controlled crossover trial to compare the effects of taking oral curcumin to those placebo on against evaluation markers of DOMS immediately after a bout of unaccustomed squat exercises. All subjects completed two test trials at an interval of at least 2 weeks. Randomization was applied to both groups (curcumin and placebo treatments). Exclusion criteria comprised regular leg weight training in the 3 months prior to the tests; current lower limb musculoskeletal injury; current use of non-steroidal anti-inflammatory drugs and antioxidant supplement, as well as neurological disease involving the lower limb.

Dietary. Participants were instructed verbally and in writing to follow an average polyphenolcontain diet throughout the duration of participation in the study starting 3 weeks prior to the start of the study period until the last blood sampling. A list of high polyphenol-contain foods was provided to participants to help them avoid major sources of dietary polyphenols.

Induction of delayed-onset muscle soreness. DOMS was induced in all subjects by standardized repetitive quadriceps muscle exercise using a squat machine. The researchers selected one repeated maximum (1RM) on the squat machine to start with a low but reasonable weight that then increased with each repetition until subjects reached their 1RM. 1RM is defined as the highest weight under which a subject is able to perform a squat position just for one repetition.

For the main trials, subjects performed exercises (7 sets of 20 squats) using a squat machine at under 50\% 1RM. Each repetition was performed through the full pain-free range of motion in a slow controlled manner. Subjects performed the concentric portion of the repetition for 2 seconds, paused at full contraction for 1 second and then completed the eccentric portion over a 4-second period (for a total of 7 seconds per repetition). Subjects performed each set according to their level of tolerance and were given a rest period of 1 to 3 minutes between sets (25).

Intervention. Each participant underwent 2 laboratory-based trials in randomized order: (1) placebo (control), (2) curcumin supplementation. There was an interval of at least 2 weeks 
between trials. Curcumin was provided by the Thera values Corporation design (Tokyo, Japan) (26). The product was developed to result in much higher plasma concentration and bioavailability after intake compared to conventional curcumin powder. The curcumin capsule in this study consisted of $10 \%$ curcumin, $2 \%$ curcuminoids without curcumin, $3.2 \%$ gum ghatti, $0.27 \%$ citric acid, $54.53 \%$ dextrin and $30 \%$ maltose. The placebo capsule consisted of $5 \%$ tartrazine, $3.5 \%$ gum ghatti, $0.3 \%$ citric acid, $59.2 \%$ dextrin and $32 \%$ maltose. Participants received oral administration of $150 \mathrm{mg}$ curcumin or the same capsules of placebo from the researchers after exercise (just after second blood sampling)(27).

Measurement of muscle damage and oxidative stress markers. Muscle damage and oxidative stress markers were measured immediately before, after, 24,48 , and $72 \mathrm{~h}$ after eccentric strength exercise. Muscle soreness was assessed using a visual analogue pain scale (VAS), for which participants placed a mark on a $10 \mathrm{~cm}$ line to indicate a degree of soreness. Soreness was measured by distance in centimeters from the left end of the scale to the mark. Establishment of validity and reliability of VAS as a measure for subjective soreness was cited in another report (28). Markers for muscle damage were as follows; , creatine kinase (CK), alanine aminotransferase (ALT), and aspartate aminotransferase (AST); measured using spectrophotometer commercial kits (ParsAzmoon, Iran). Total antioxidant capacity (TAC) was used to mark oxidative stress and measured by the method cited in Varga et al. (29).

Statistical Analysis. All data are presented as mean \pm SEM, and statistical significance was set at $\mathrm{p}<0.05$. Data with multiple time points during the main trial were analyzed using the mixedmodel repeated-measures ANOVA. Mauchly's test was consulted and Greenhouse-Geisser correction was applied if the sphericity assumption was violated. If a significant $p$ value was identified for the main effect of time (time of sample), multiple pair wise comparison was made using Bonferroni confidence interval adjustment. Moreover, comparison was made of dependent variable data in multiple time points between the two groups using the independent sample t-test.

\section{RESULTS}

Physical characteristics of the participants were as follows (expressed as mean \pm standard deviation [SD]): age, $25.0 \pm 1.6$ years; height, $178.9 \pm 4.1$ $\mathrm{cm}$; body mass, $81.1 \pm 6.8 \mathrm{~kg}$; fat $\%, 14.2 \pm 2.1$.

Total Antioxidant Capacity (TAC). Comparison of baseline resting plasma TAC showed no difference between the groups $(\mathrm{P}>0.05)$. TAC was significantly higher in $\mathrm{C}$ group than in group $\mathrm{P}$ at 24 and $48 \mathrm{~h}$ after exercise $(\mathrm{P}=0.001$ and $\mathrm{P}=0.01$, respectively) (Figure 1).

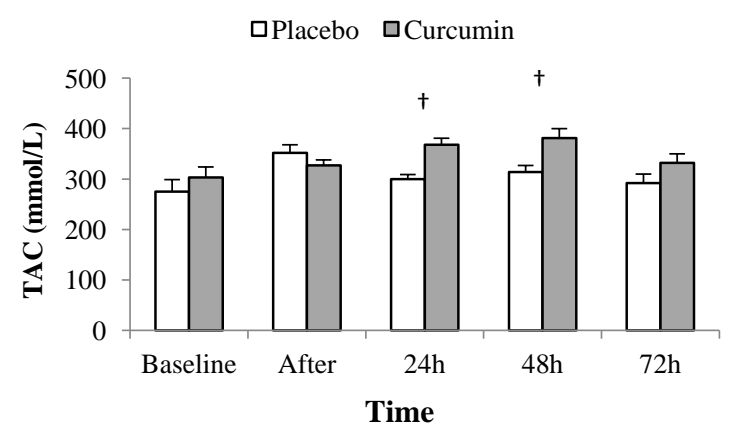

Figure 1. TAC plasma level. Values represent means \pm SEM. $\uparrow$ demonstrate a significant increase in group $\mathrm{C}$ compared with group $\mathrm{P}(\mathrm{p}<0.05)$.

CK. Baseline resting serum $\mathrm{CK}$ showed no difference different between groups $\mathrm{C}$ and $\mathrm{P}$ $(\mathrm{P}>0.05)$. CK showed a significant increase immediately, and at $72 \mathrm{~h}$ after exercise in both groups compared with pre-exercise $(\mathrm{P}<0.05)$. CK was significantly lower in $\mathrm{C}$ group compared with group P 24h after exercise ( $\mathrm{P}=0.019)$ (Figure 2).

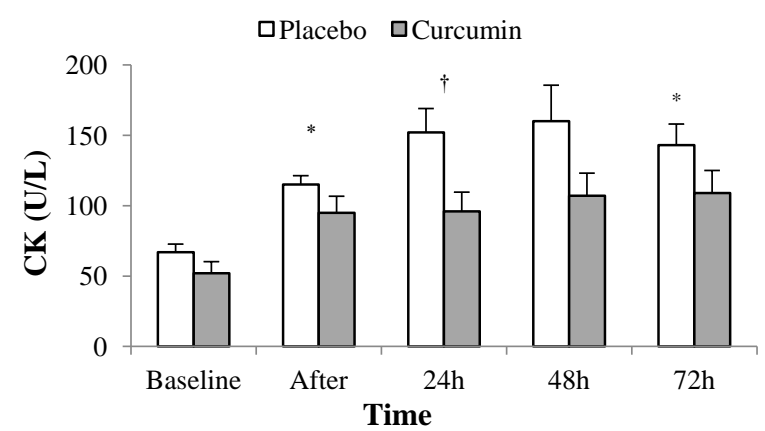

Figure 2. CK before and after exercise. Values represent means \pm SEM. $*$ show significant increase compared with pre-exercise in both groups $(\mathrm{p}<0.05)$. $\dagger$ demonstrates a significant increase compared with pre-exercise only in group $\mathrm{P}$ and a significant increase in group $\mathrm{P}$ compared with group $\mathrm{C}(\mathrm{p}<0.05)$. 
Visual Analogue Scale (VAS). VAS showed significant increase at the following times; immediately, 24, 48, and 72h after exercise in both groups compared with pre-exercise $(\mathrm{P}<0.05)$. VAS was significantly lower in group $\mathrm{C}$ compared with group $\mathrm{P}$ at the times; 48 , and $72 \mathrm{~h}$ after exercise $(\mathrm{P}<0.05)$ (Figure 3$)$.

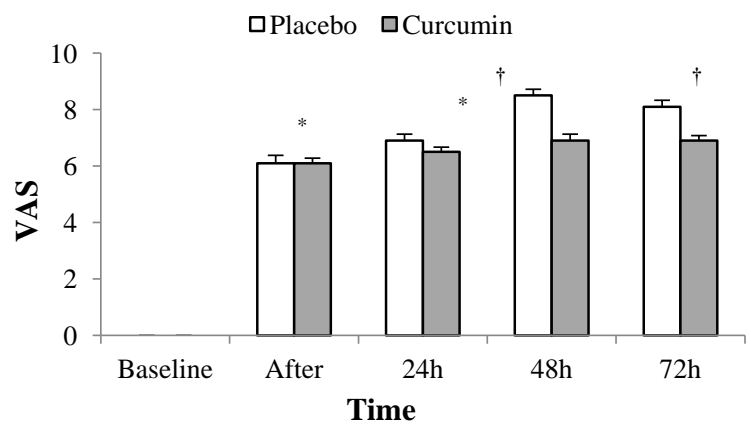

Figure 3. VAS before and after exercise. Values represent means \pm SEM. * shows significant increase compared with pre-exercise in both groups $(\mathrm{p}<0.05)$. $\dagger$ demonstrates a significant increase compared with pre-exercise in both groups and significant increase in group $\mathrm{P}$ compared with group $\mathrm{C}(\mathrm{p}<0.05)$.
ALT and AST. Baseline resting serum ALT and AST were not different between groups $(\mathrm{P}>0.05)$. ALT significantly increased immediately, and $24 \mathrm{~h}$ after exercise in both groups $(\mathrm{P}<0.05)$. ALT was significantly lower in $\mathrm{C}$ group compared with the $\mathrm{P}$ group $24 \mathrm{~h}$ after exercise $(\mathrm{P}=0.019)$ (Table 2). AST was significantly lower in $\mathrm{C}$ group compared with the $\mathrm{P}$ group just $24 \mathrm{~h}$ after exercise $(\mathrm{P}=0.034)$ (Table 1).

\section{DISCUSSION}

To the best of our knowledge, the present study was the first to examine the effects of $150 \mathrm{mg}$ curcumin supplement on antioxidant capacity and muscle damage markers after intensive eccentric exercise in humans. The main findings in this study were as follows: (1) curcumin supplement maintained TAC high, 24 and $48 \mathrm{~h}$ after exercise (2) It enabled attenuation of CK, LDH, ALT, AST, and experience of pain related to DOMS.

Table 1. ALT and AST before and after exercise. Values represent means \pm SEM.

\begin{tabular}{ccccccc}
\hline & Groups & Baseline & $\begin{array}{c}\text { Immediately } \\
\text { after Exercise }\end{array}$ & $\begin{array}{c}\text { 24h after } \\
\text { Exercise }\end{array}$ & $\begin{array}{c}\text { 48h after } \\
\text { Exercise }\end{array}$ & $\begin{array}{c}\text { 72h after } \\
\text { Exercise }\end{array}$ \\
\hline ALT & Placebo & $17.4 \pm 0.62$ & $21.0 \pm 1.16^{*}$ & $25.5 \pm 1.73^{\dagger}$ & $23.0 \pm 1.76$ & $20.1 \pm 1.97$ \\
(U/L) & Curcumin & $17.7 \pm 0.61$ & $22.9 \pm 1.48^{*}$ & $20.4 \pm 0.90^{*}$ & $19.8 \pm 0.91$ & $18.2 \pm 1.34$ \\
\hline AST & Placebo & $21.2 \pm 2.32$ & $24.4 \pm 1.53$ & $31.1 \pm 2.21^{\dagger}$ & $23.0 \pm 1.30$ & $24.4 \pm 1.51$ \\
(U/L) & Curcumin & $20.9 \pm 0.81$ & $21.3 \pm 1.11$ & $24.0 \pm 2.23$ & $26.6 \pm 1.93$ & $26.6 \pm 1.89$ \\
\hline
\end{tabular}

* shows significant increase compared with pre-exercise $(\mathrm{p}<0.05)$.

$\uparrow$ demonstrates a significant increase compared with pre-exercise in both groups and significant increase in group $\mathrm{P}$ compared with group $\mathrm{C}(\mathrm{p}<0.05)$.

It is known that Curcumin has antioxidant quality (18). According to the findings of this study, TAC remained significantly higher in group $\mathrm{C}$ than in group $\mathrm{P}$ at 24 and $48 \mathrm{~h}$ after exercise; this can possibly be attributed to antioxidant properties of curcumin (Figure 1). In parallel, markers of muscle damage enzyme showed a reduction in group $\mathrm{C}$ group compared to group P. Observations shown in figures 2, 3, and Table 1, alteration patterns of CK, ALT, and AST were almost the same as TAC. CK, and ALT were significantly enhanced after exercise demonstrating occurrence of DOMS, but there was a significant reduction at some points of the time series in group C. One explanation is that the antioxidant properties of curcumin caused a decline in some enzymes.

Elevations of CK and ALT serum levels show increased leakage of these enzymes through cell membranes after exercise. Oxidative stress induced by lipid peroxidation may lead to membrane permeability that would allow muscle constituents such as $\mathrm{CK}$ and aminotransferases to escape (30). The phenolic compound of curcumin acts as a scavenger of reactive oxygen species and a quencher of the lipid peroxidative side chain (31). Thus, the phenolic $\mathrm{OH}$ groups of curcumin may have reduced lipid hydroperoxides. Finally, the inhibitory effects of curcumin on lipid peroxidation may have 
prevented CK leakage and other related enzymes from cell membranes and consequently less increase in CK serum level. Previous studies have confirmed that supplements containing antioxidants are able to attenuate these enzymes $(32,33)$.

Attenuation of these enzymes could also be attributed to the anti-inflammatory effects of curcumin. Several studies have confirmed that curcumin blocks the activity of transcription factor NF-kappaB, reduces AP-1 binding to DNA and causes decreased production of COX2 , all of which play a role in inflammation (22, $34,35)$. Curcumin appears to target NF- kappaB as opposed to COX-2, indicating the potential for less serious side effects than NSAIDs (22). This mechanism could also be responsible for reduced inflammatory response to exercise observed in this study.

Some investigations have evaluated the analgesic efficacy of curcumin. Di Pierro et al. reports that $400 \mathrm{mg}$ curcumin had a well-defined pain-relieving effect, even greater than that of acetaminophen $500 \mathrm{mg}$, and was better tolerated than nimesulide (36). Drobnic et al. also shows that curcumin supplement caused a decrease in DOMS-induced pain (23). The findings of this study support those results of earlier research. According to our data, $150 \mathrm{mg}$ curcumin supplement resulted in pain reduction of 48 and
$72 \mathrm{~h}$ after exercise (Figure 4). This acute effect was probably related to desensitization or inhibition of a series of transient receptor potential ion channels involved in the generation of painful stimuli such as TRPV1 and TRPA1 $(37,38)$.

\section{CONCLUSION}

The results of this study demonstrate that acute curcumin supplement after intensive eccentric exercise can not only keep antioxidant capacity responses high, but also serve to decrease muscle damage and pain in humans.

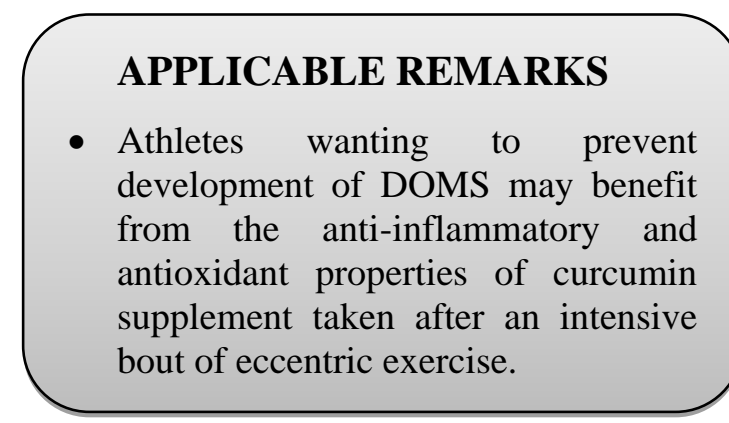

\section{ACKNOWLEDGMENTS}

The authors wish to thank Ardabil branch, Islamic Azad University for supplying technical and instrumental assistance.

\section{REFERENCES}

1. Pournot H, Bieuzen F, Louis J, Fillard J-R, Barbiche E, Hausswirth C. Time-course of changes in inflammatory response after whole-body cryotherapy multi exposures following severe exercise. PloS one. 2011;6(7):e22748.

2. Arent SM, Senso M, Golem DL, McKeever KH. The effects of theaflavin-enriched black tea extract on muscle soreness, oxidative stress, inflammation, and endocrine responses to acute anaerobic interval training: a randomized, double-blind, crossover study. Journal of the International Society of Sports Nutrition. 2010;7(11).

3. Kanda K, Sugama K, Hayashida H, Sakuma J, Kawakami Y, Miura S, et al. Eccentric exercise-induced delayedonset muscle soreness and changes in markers of muscle damage and inflammation. Exerc Immunol Rev. 2013;19(19):72-85.

4. Hoseinzadeh K, Daryanoosh F, Baghdasar PJ, Alizadeh H. Acute effects of ginger extract on biochemical and functional symptoms of delayed onset muscle soreness. Medical Journal of The Islamic Republic of Iran. 2015;29:261.

5. Gatterer H, Peters P, Philippe M, Burtscher M. The effect of pulsating electrostatic field application on the development of delayed onset of muscle soreness (DOMS) symptoms after eccentric exercise. Journal of physical therapy science. 2015;27(10):3105.

6. Kingsley MI, Kilduff LP, McEneny J, Dietzig RE, Benton D. Phosphatidylserine supplementation and recovery following downhill running. Medicine and science in sports and exercise. 2006;38(9):1617-25.

7. Kim J, Lee J. A review of nutritional intervention on delayed onset muscle soreness. Part I. Journal of exercise rehabilitation. 2014;10(6):349-56.

8. da Silva LA, Tromm CB, Bom KF, Mariano I, Pozzi B, da Rosa GL, et al. Effects of taurine supplementation following eccentric exercise in young adults. Applied Physiology, Nutrition, and Metabolism. 2013;39(1):101-4. 
9. Hurley CF, Hatfield DL, Riebe DA. The effect of caffeine ingestion on delayed onset muscle soreness. The Journal of Strength \& Conditioning Research. 2013;27(11):3101-9.

10. Tartibian B, Maleki BH, Abbasi A. Omega-3 fatty acids supplementation attenuates inflammatory markers after eccentric exercise in untrained men. Clinical Journal of Sport Medicine. 2011;21(2):131-7.

11. Malaguti M, Angeloni C, Hrelia S. Polyphenols in Exercise Performance and Prevention of Exercise-Induced Muscle Damage. Oxidative medicine and cellular longevity. 2013;2013.

12. Pandey KB, Rizvi SI. Plant polyphenols as dietary antioxidants in human health and disease. Oxidative medicine and cellular longevity. 2009;2(5):270-8.

13. Aggarwal BB, Sundaram C, Malani N, Ichikawa H. Curcumin: the Indian solid gold. The molecular targets and therapeutic uses of curcumin in health and disease: Springer; 2007. p. 1-75.

14. Aggarwal BB, Gupta SC, Sung B. Curcumin: an orally bioavailable blocker of TNF and other pro-inflammatory biomarkers. British journal of pharmacology. 2013;169(8):1672-92.

15. Gao Y, Shi Q, Xu S, Du C, Liang L, Wu K, et al. Curcumin promotes KLF5 proteasome degradation through downregulating YAP/TAZ in bladder cancer cells. International journal of molecular sciences. 2014;15(9):15173-87.

16. Soetikno V, Sari FR, Sukumaran V, Lakshmanan AP, Harima M, Suzuki K, et al. Curcumin decreases renal triglyceride accumulation through AMPK-SREBP signaling pathway in streptozotocin-induced type 1 diabetic rats. The Journal of nutritional biochemistry. 2013;24(5):796-802.

17. Ramamoorthi G, Sivalingam N. Molecular mechanism of TGF- $\beta$ signaling pathway in colon carcinogenesis and status of curcumin as chemopreventive strategy. Tumor Biology. 2014;35(8):7295-305.

18. Avci G, Kadioglu H, Sehirli AO, Bozkurt S, Guclu O, Arslan E, et al. Curcumin protects against ischemia/reperfusion injury in rat skeletal muscle. Journal of Surgical Research. 2012;172(1):e39-e46.

19. Graumlich JF. Preventing gastrointestinal complications of NSAIDs: risk factors, recent advances, and latest strategies. Postgraduate medicine. 2001;109(5):117-28.

20. Mukherjee D, Nissen SE, Topol EJ. Risk of cardiovascular events associated with selective COX-2 inhibitors. Jama. 2001;286(8):954-9.

21. Nicol LM, Rowlands DS, Fazakerly R, Kellett J. Curcumin supplementation likely attenuates delayed onset muscle soreness (DOMS). European journal of applied physiology. 2015;115(8):1769-77.

22. Davis JM, Murphy EA, Carmichael MD, Zielinski MR, Groschwitz CM, Brown AS, et al. Curcumin effects on inflammation and performance recovery following eccentric exercise-induced muscle damage. American Journal of Physiology-Regulatory, Integrative and Comparative Physiology. 2007;292(6):R2168-R73.

23. Drobnic F, Riera J, Appendino G, Togni S, Franceschi F, Valle X, et al. Reduction of delayed onset muscle soreness by a novel curcumin delivery system $\left(\right.$ Meriva $\left.{ }^{\circledR}\right)$ : a randomised, placebo-controlled trial. J Int Soc Sports Nutr. 2014;11(31):10.1186.

24. Huang W-C, Chiu W-C, Chuang H-L, Tang D-W, Lee Z-M, Wei L, et al. Effect of curcumin supplementation on physiological fatigue and physical performance in mice. Nutrients. 2015;7(2):905-21.

25. Shimomura Y, Kobayashi H, Mawatari K, Akita K, Inaguma A, Watanabe S, et al. Effects of squat exercise and branched-chain amino acid supplementation on plasma free amino acid concentrations in young women. Journal of nutritional science and vitaminology. 2009;55(3):288-91.

26. Takahashi M, Suzuki K, Kim H, Otsuka Y, Imaizumi A, Miyashita M, et al. Effects of curcumin supplementation on exercise-induced oxidative stress in humans. International journal of sports medicine. 2014;35(6):469-75.

27. Tanabe Y, Maeda S, Akazawa N, Zempo-Miyaki A, Choi Y, Ra S-G, et al. Attenuation of indirect markers of eccentric exercise-induced muscle damage by curcumin. European journal of applied physiology. 2015;115(9):1949-57.

28. Gallagher EJ, Bijur PE, Latimer C, Silver W. Reliability and validity of a visual analog scale for acute abdominal pain in the ED. The American journal of emergency medicine. 2002;20(4):287-90.

29. Varga IS, Matkovics B. Comparative study of plasma antioxidant status in normal and pathological cases. Pathophysiology. 1998;5:77.

30. Armstrong R, Warren G, Warren J. Mechanisms of exercise-induced muscle fibre injury. Sports Medicine. 1991;12(3):184-207.

31. Singh U, Barik A, Singh BG, Priyadarsini KI. Reactions of reactive oxygen species (ROS) with curcumin analogues: Structure-activity relationship. Free radical research. 2011;45(3):317-25.

32. Bohlooli S, Barmaki S, Khoshkhahesh F, Nakhostin-Roohi B. The effect of spinach supplementation on exerciseinduced oxidative stress. The Journal of sports medicine and physical fitness. 2015;55(6):609-14.

33. Nakhostin-Roohi B, Khoshkhahesh F, Parandak K, Ramazanzadeh R. Influence of L-carnitine supplementation on exercise-induced muscle damage. Medicina Dello Sport. 2014;67(2):251-9. 
34. Singh S, Aggarwal BB. Activation of transcription factor NF- $\mathrm{BB}$ is suppressed by curcumin (diferuloylmethane). Journal of Biological Chemistry. 1995;270(42):24995-5000.

35. Thaloor D, Miller KJ, Gephart J, Mitchell PO, Pavlath GK. Systemic administration of the NF-kB inhibitor curcumin stimulates muscle regeneration after traumatic injury. American Journal of Physiology-Cell Physiology. 1999;277(2):C320-C9.

36. Di Pierro F, Rapacioli G, Di Maio E, Appendino G, Franceschi F, Togni S. Comparative evaluation of the painrelieving properties of a lecithinized formulation of curcumin (Meriva (®)), nimesulide, and acetaminophen. J Pain Res. 2013;6:201-5.

37. Leamy AW, Shukla P, McAlexander MA, Carr MJ, Ghatta S. Curcumin ((E, E)-1, 7-bis (4-hydroxy-3methoxyphenyl)-1, 6-heptadiene-3, 5-dione) activates and desensitizes the nociceptor ion channel TRPA1. Neuroscience letters. 2011;503(3):157-62.

38. Yeon K, Kim S, Kim Y, Lee M, Ahn D, Kim H, et al. Curcumin produces an antihyperalgesic effect via antagonism of TRPV1. Journal of dental research. 2010;89(2):170-4. 